\title{
Select Biomarkers on the Day of Anterior Cruciate Ligament Reconstruction Predict Poor Patient-Reported Outcomes at 2-Year Follow-Up: A Pilot Study
}

\author{
Christian Lattermann, ${ }^{1}$ Caitlin E.-W. Conley, ${ }^{2}$ Darren L. Johnson, ${ }^{2}$ \\ Emily K. Reinke, ${ }^{3}$ Laura J. Huston $\left(\mathbb{D},{ }^{3}\right.$ Janet L. Huebner, ${ }^{4}$ Ching-Heng Chou, ${ }^{4}$ \\ Virginia B. Kraus, ${ }^{4}$ Kurt P. Spindler, ${ }^{5}$ and Cale A. Jacobs $(\mathbb{D})^{2}$ \\ ${ }^{1}$ Brigham and Women's Hospital, Harvard Medical School, Chestnut Hill, MA, USA \\ ${ }^{2}$ University of Kentucky, Lexington, KY, USA \\ ${ }^{3}$ Vanderbilt University, Nashville, TN, USA \\ ${ }^{4}$ Duke University, Durham, NC, USA \\ ${ }^{5}$ Cleveland Clinic Sports Health Center, Garfield Heights, OH, USA
}

Correspondence should be addressed to Cale A. Jacobs; cale.jacobs@uky.edu

Received 7 March 2018; Revised 5 June 2018; Accepted 4 July 2018; Published 19 July 2018

Academic Editor: Jiang Du

Copyright ( 2018 Christian Lattermann et al. This is an open access article distributed under the Creative Commons Attribution License, which permits unrestricted use, distribution, and reproduction in any medium, provided the original work is properly cited.

Background. The majority of patients develop posttraumatic osteoarthritis within 15 years of anterior cruciate ligament (ACL) injury. Inflammatory and chondrodegenerative biomarkers have been associated with both pain and the progression of osteoarthritis; however, it remains unclear if preoperative biomarkers differ for patients with inferior postoperative outcomes. Hypothesis/Purpose. The purpose of this pilot study was to compare biomarkers collected on the day of ACL reconstruction between patients with "good" or "poor" 2-year postoperative outcomes. We hypothesized that inflammatory cytokines and chondrodegenerative biomarker concentrations would be significantly greater in patients with poorer outcomes. Study Design. Prospective cohort design. Methods. 22 patients ( 9 females, 13 males; age $=19.5 \pm 4.1$ years; $\mathrm{BMI}=24.1 \pm 3.6 \mathrm{~kg} / \mathrm{m}^{2}$ ) previously enrolled in a randomized trial evaluating early anti-inflammatory treatment after ACL injury. Biomarkers of chondrodegeneration and inflammation were assessed from synovial fluid (sf) samples collected on the day of ACL reconstruction. Participants completed Knee Injury and Osteoarthritis Outcome Score (KOOS) and International Knee Documentation Committee (IKDC) questionnaires two years following surgery. Patients were then categorized based on whether their KOOS Quality of Life (QOL) score surpassed the Patient Acceptable Symptom State (PASS) threshold of 62.5 points or the IKDC PASS threshold of 75.9 points. Results. Patients that failed to reach the QOL PASS threshold after surgery $(\mathrm{n}=6,27 \%)$ had significantly greater sf interleukin-1 alpha (IL- $1 \alpha$; $\mathrm{p}=0.004)$, IL-1 receptor antagonist (IL-1ra; $\mathrm{p}=0.03$ ), and matrix metalloproteinase-9 (MMP-9; $\mathrm{p}=0.01)$ concentrations on the day of surgery. Patients that failed to reach the IKDC PASS threshold $(\mathrm{n}=9,41 \%)$ had significantly greater sf IL- $1 \alpha(\mathrm{p}=0.02)$. Conclusion. These pilot data suggest that initial biochemical changes after injury may be an indicator of poor outcomes that are not mitigated by surgical stabilization alone. Biological adjuvant treatment in addition to ACL reconstruction may be beneficial; however, these data should be used for hypothesis generation and more definitive randomized clinical trials are necessary.

\section{Introduction}

Whether isolated or in concert with concomitant meniscal or articular cartilage injury, anterior cruciate ligament (ACL) rupture initiates a cascade of cytokine and catabolic enzyme activity [1-4]. Regardless of surgical or conservative management, the majority of patients develop posttraumatic osteoarthritis (PTOA) within 15 years of ACL injury [5-7]. In addition to magnetic resonance imaging (MRI) and/or radiographic evidence of cartilage degeneration [8-11], the 
TABLE 1: Concomitant injuries and surgical treatments (n (\%)) performed at the time of ACL reconstruction.

\begin{tabular}{|c|c|c|c|c|}
\hline & Not injured & Injured, not treated & Debrided & Repaired \\
\hline \multicolumn{5}{|l|}{ Meniscus } \\
\hline Medial & $8(36 \%)$ & $0(0 \%)$ & $1(5 \%)$ & $13(59 \%)$ \\
\hline Lateral & $8(36 \%)$ & $0(0 \%)$ & $3(14 \%)$ & $11(50 \%)$ \\
\hline \multicolumn{5}{|c|}{ Tibial Articular Cartilage } \\
\hline Medial & $22(100 \%)$ & $0(0 \%)$ & $0(0 \%)$ & $0(0 \%)$ \\
\hline Lateral & $22(100 \%)$ & $0(0 \%)$ & $0(0 \%)$ & $0(0 \%)$ \\
\hline \multicolumn{5}{|c|}{ Femoral Articular Cartilage } \\
\hline Medial & $21(95 \%)$ & $1(5 \%)$ & $0(0 \%)$ & $0(0 \%)$ \\
\hline Lateral & $22(100 \%)$ & $0(0 \%)$ & $0(0 \%)$ & $0(0 \%)$ \\
\hline
\end{tabular}

onset of PTOA is associated with increased concentrations of chondrodegenerative and inflammatory biomarkers [12, 13]. Increased biomarkers of cartilage turnover have not only been reported as a late consequence of ACL injury, but also been reported to be significantly elevated immediately following ACL injury $[2-4,14]$. This may indicate chronically increased cartilage turnover and a progressively destructive process that may have long-term clinical consequences. However, while inflammatory and chondrodegenerative biomarkers are elevated in the period between ACL injury and reconstruction $[3,4]$, there is a substantial knowledge gap with regard to the role of preoperative biomarker concentrations and their association with either successful or suboptimal clinical outcomes.

One method to objectively quantify a successful outcome is to determine whether a patient has achieved a Patient Acceptable Symptom State (PASS) following surgery [15-18]. The PASS represents a threshold for a given postoperative outcome score above which the patient is defined as having a satisfactory outcome. Patients that fail to achieve the PASS threshold are then considered to have a suboptimal postoperative outcome. The purpose of this pilot study was to compare biomarkers assessed from samples collected preoperatively on the day of ACL reconstruction between patients with 2year postoperative patient-reported outcome scores above and below previously established PASS thresholds [18]. We hypothesized that synovial fluid and urine concentrations of inflammatory cytokines and chondrodegenerative biomarkers would be significantly greater in patients with poorer outcomes.

\section{Methods}

Participants included 22 patients ( 9 females, 13 males; age= $19.5 \pm 4.1$ years (mean \pm standard deviation); $\mathrm{BMI}=$ $24.1 \pm 3.6 \mathrm{~kg} / \mathrm{m}^{2}$, Table 1 ) that had previously consented to participate at one site of an IRB-approved, multicenter prospective randomized clinical trial evaluating early antiinflammatory treatment after ACL injury (clinicaltrials.gov ID: NCT01692756) [3]. At the time of enrollment, patients with an isolated ACL injury between the ages of 14 and 32 with closed growth plates were included. Other inclusion criteria included a normal contralateral knee, no history of previous traumatic ipsilateral knee injury, and an ACL injury that occurred during sports activity with no clinical evidence of posterior cruciate ligament injury and with no more than grade 1 medial or lateral collateral ligament injury. Patients were excluded if the injury occurred more than eight days prior to enrollment or they had a history of previous ipsilateral knee surgery, a known allergy to triamcinolone acetonide, intra-articular cortisone injection into either knee within three months of injury, or a history of any inflammatory disease or immunocompromise [3].

In the initial trial, biomarkers were assessed at three time points prior to surgery to determine if early anti-inflammatory treatment could alter the expected progressive increases in inflammatory and chondrodegenerative biomarker concentrations [3]. The three time points from the initial study were (1) mean 4 days after injury, (2) mean 11 days after injury, and (3) the day of ACL reconstruction (mean 37 days after injury). For the current study, we used only biomarker data from samples acquired on the day of ACL reconstruction to assess if inflammatory and chondrodegenerative biomarker concentrations differed between those with good versus poor postoperative outcomes. In original study, patient-reported outcomes on the day of surgery did not differ between patients treated with preoperative intra-articular corticosteroid and the placebo group. In addition, the surgical intervention and postoperative rehabilitation and medications did not differ between groups and no between-group differences were noted in either postoperative Knee Injury and Osteoarthritis Outcome Score (KOOS) Quality of Life scores (QOL; corticosteroid = 72.7 \pm 16.1 versus placebo $=67.8 \pm 31.1, \mathrm{p}=0.82)$ or International Knee Documentation Committee scores (IKDC; corticosteroid = $74.8 \pm 12.9$ versus placebo $=71.2 \pm 12.3, \mathrm{p}=0.46)$. As such, we therefore pooled data for all patients for the current analysis.

Knee aspiration was performed aseptically at the time of surgery via a superolateral suprapatellar approach, and intra-articular placement was confirmed using the "squish" test [19]. Patients were fasting as both urine and synovial fluid (sf) samples were collected on the morning of surgery. Synovial fluid and urine samples were spun at 3500 RPM for 10 minutes and the supernatant aliquoted according to an allotment protocol. Once all samples were collected they were shipped to the Duke University biomarker laboratory (VBK/JLH) for analysis [3]. 
A number of chondrodegenerative biomarkers and inflammatory cytokines were assessed using previously described methods (Table 2) [3, 4]. All biomarker concentrations were assessed from synovial fluid samples with the exception of CTX-II which was assessed in both the synovial fluid (CTX-II) and urine (uCTX-II). Urinary CTX-II was normalized to creatinine concentration (ng/mmol) [13]. Biomarker variability, linear range of standard, and need for repeat were carefully assessed. Several commerciallyavailable ELISA kits provided controls, which were used with every run to confirm that values were within the acceptable range according to the manufacturer and to assess interassay (plate to plate) variability. In addition, synovial fluid from a human subject with knee OA that had previously been aliquoted and frozen at $-80^{\circ} \mathrm{C}$ was used as the control for all assays. A fresh aliquot of this control synovial fluid was thawed and used on every plate to calculate intraand interassay variance of the assay each day that analyses were performed. Due to limited sf sample volume available, samples were run in singlicate; however, all standards and controls were run in duplicate and used to determine the precision of the assay and to establish an acceptable control range for the assay. The mean of the control sample for all assays plus or minus two standard deviations was defined as the acceptable control range. All control samples were within the acceptable control range for all plates and assays. Mean intra-assay coefficients of variation for each assay are reported in Table 2. Mean interassay coefficients of variation were all $<15 \%$ with the exception of IL- $1 \alpha$ and IL- $1 \beta$. The manufacturer reported that interassay CVs for these assays are $6.6 \%$ and $6.4 \%$, respectively; however due to the human control SF sample having values at or below the level of detection, this calculation was not possible. For values of IL$1 \alpha$ and IL- $1 \beta$ that were below the lower limit of detection, $1 / 2$ LLOD (lowest level of detection) was reported for statistical purposes.

Patients were contacted two years after surgery to complete standardized patient-reported outcomes, including IKDC and KOOS Scores [18, 21-25]. The volume of preoperative bone bruises was calculated from each patient's preoperative magnetic resonance imaging (MRI) scan using previously described methods [26]. MRIs were considered adequate for inclusion if they included T2 or PD sequences in the coronal, axial, and sagittal plane as well as slice thicknesses < $5 \mathrm{~mm}$. The bone bruise volumes in the medial tibial plateau, medial femoral condyle, lateral tibial plateau, and lateral femoral condyle were measured from the T2 or PD weighted coronal images in a modification of Roemer and Bohndorf's technique $[26,27]$. Bone bruises volumes from each of the four bony regions were then summed and expressed as the total bone bruise volume $\left(\mathrm{mm}^{3}\right)$ [26].

2.1. Statistical Analysis. Mann-Whitney U tests were performed to determine if inflammatory cytokines and chondrodegenerative biomarkers assessed from samples collected on the day of surgery (Table 2) differed from those with either a good or poor outcome based on postoperative IKDC and KOOS QOL scores. Due to the high number of IL-1 $\beta$ samples that were below the lower limits of detection (11/22, $50 \%$ ), for this cytokine we also used Fisher Exact tests to compare the prevalence of concentrations below the lower limits of detection (LLOD) between groups. Patients were categorized as having a good or poor outcome based on whether they reported IKDC or KOOS QOL scores above or below previously established Patient Acceptable Symptom State (PASS) thresholds following ACL reconstruction of 62.5 points for KOOS QOL and 75.9 points for the IKDC [18]. As the KOOS QOL and IKDC may be assessing different aspects of the patient's outcome, separate analyses were run based on the KOOS QOL PASS definition and IKDC PASS definition. Cohen's d effect sizes calculations were also performed in order to identify potentially clinically-meaningful findings within these pilot data, with $\mathrm{d}>0.80$ considered a large effect size [28]. Analyses were performed using SPSS Statistics 24 (IBM, Armonk, NY) and Excel 2016 (Microsoft, Redmond, WA) with $\mathrm{p}<0.05$ considered statistically significant. All data and results are stored at the Department of Orthopaedic Surgery and Sports Medicine at the University of Kentucky.

\section{Results}

At a mean follow-up of 2.4 years, 6/22 patients (27\%) had postoperative KOOS QOL scores below the PASS threshold of 62.5 points (Table 3 ). Patients that failed to reach the QOL PASS threshold had significantly greater sf IL- $1 \alpha(\mathrm{p}=0.004)$, sf IL-1ra $(p=0.02)$, and sf MMP-9 $(p=0.01)$. While not statistically significant, markers of type I and type II collagen breakdown (sf NTX-I [p=0.055] and uCTX-II [p=0.08]) tended to be greater for those that failed to reach the QOL PASS threshold. Graft type, the prevalence of meniscus injury, and bone bruise volumes did not differ between QOL PASS groups (Table 3 ).

Nine of 22 patients (41\%) failed to reach the IKDC PASS threshold of 75.9 (Table 4). Patients that failed to reach the IKDC PASS threshold had significantly greater sf IL- $1 \alpha(\mathrm{p}=0.02)$. While not statistically significant, sf IL1ra $(\mathrm{p}=0.057)$, sf MMP-1 $(\mathrm{p}=0.10)$, and sf MMP-9 $(\mathrm{p}=0.10)$ tended to be greater for those that failed to reach the IKDC PASS threshold. No other biomarker differences were noted between those that did or did not surpass the IKDC or QOL PASS thresholds. Graft type, the prevalence of meniscus injury, and bone bruise volumes did not differ between IKDC PASS groups (Table 4).

\section{Discussion}

The purpose of this pilot study was to compare biomarkers assessed from samples collected on the day of ACL reconstruction between patients with postoperative patientreported outcome scores above and below previously established PASS thresholds [18]. We hypothesized that synovial fluid and urinary concentrations of inflammatory cytokines and chondrodegenerative biomarkers would be significantly greater in patients with poorer outcomes, and our hypotheses were, in part, supported by the current results. 


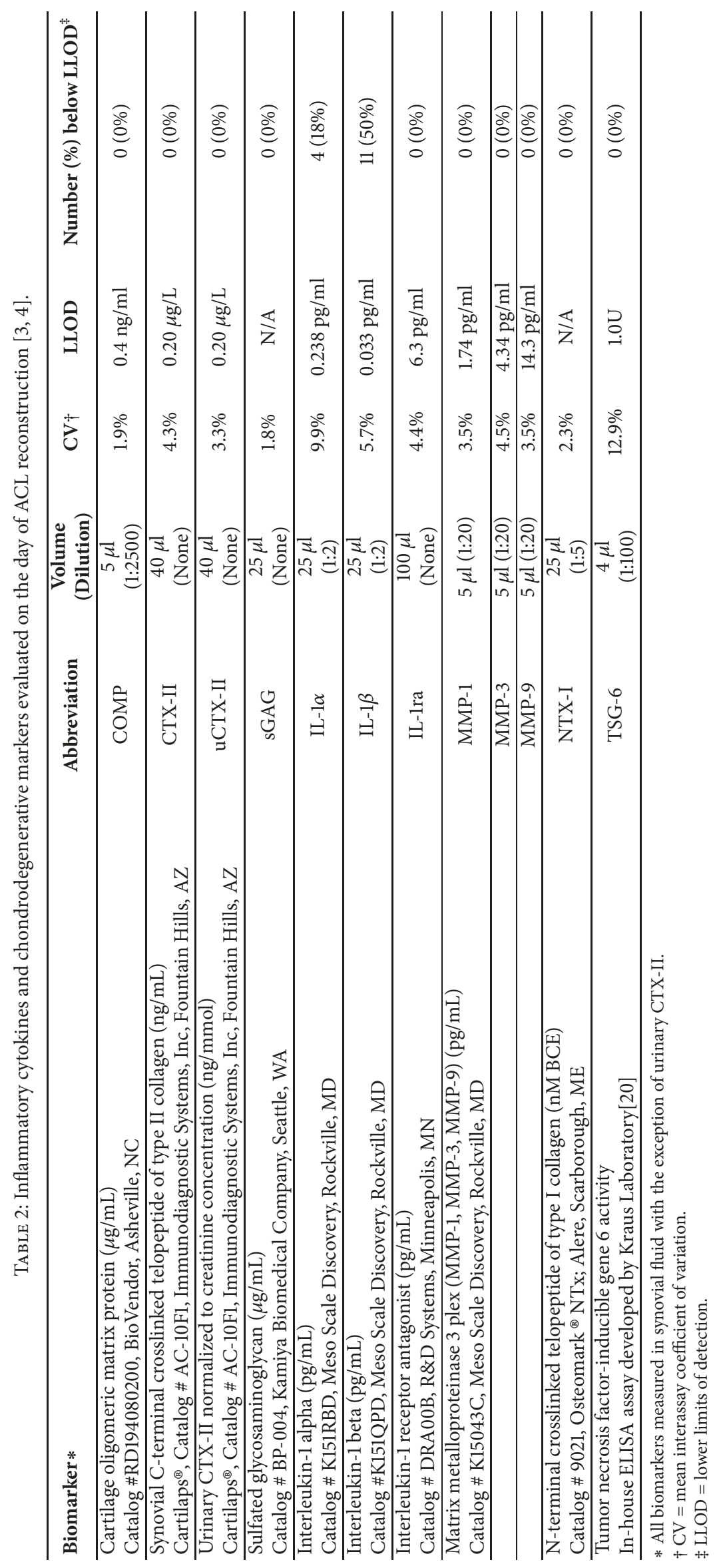


TABLE 3: Comparison of inflammatory cytokines and chondrodegenerative markers (mean \pm standard deviation) evaluated on the day of ACL reconstruction between patients with KOOS QOL scores above and below the PASS threshold of 62.5 points.

\begin{tabular}{|c|c|c|c|c|}
\hline Biomarker & $<$ PASS & $\geq$ PASS & $\mathrm{p}^{\mathrm{a}}$ & $d^{e}$ \\
\hline $\mathrm{N}$ & 6 & 16 & - & - \\
\hline Female/Male (n) & $3 / 3$ & $6 / 10$ & 0.66 & - \\
\hline Steroid/Placebo (n) & $4 / 2$ & $12 / 4$ & $>0.99$ & - \\
\hline Age (years) & $18.0 \pm 2.6$ & $20.0 \pm 4.5$ & 0.42 & - \\
\hline BMI $\left(\mathrm{kg} / \mathrm{m}^{2}\right)$ & $22.4 \pm 2.9$ & $24.8 \pm 3.6$ & 0.15 & - \\
\hline Graft (BTB/Hamstring) & $5 / 1$ & $13 / 3$ & $>0.99$ & - \\
\hline Medial meniscus injury & 5 & 9 & 0.35 & - \\
\hline Lateral meniscus injury & 2 & 12 & 0.12 & - \\
\hline Bone bruise volume $\left(\mathrm{mm}^{3}\right)$ & $7.99 \pm 8.93$ & $11.07 \pm 9.33$ & 0.50 & 0.30 \\
\hline $\operatorname{COMP}(\mu \mathrm{g} / \mathrm{ml})$ & $32.3 \pm 12.5$ & $39.3 \pm 14.0$ & 0.42 & 0.51 \\
\hline CTX-II (ng/ml) & $1.57 \pm 0.93$ & $1.52 \pm 1.97$ & 0.38 & 0.03 \\
\hline uCTX-II ${ }^{\mathrm{d}}(\mu \mathrm{g} / \mathrm{mmol})$ & $5.72 \pm 4.86$ & $2.42 \pm 2.09$ & 0.08 & 0.99 \\
\hline $\mathrm{sGAG}(\mu \mathrm{g} / \mathrm{ml})$ & $190.9 \pm 69.9$ & $264.7 \pm 168.3$ & 0.83 & 0.49 \\
\hline$I L-1 \alpha(p g / m l)$ & $9.47 \pm 7.65$ & $2.21 \pm 2.20$ & 0.004 & 1.36 \\
\hline $\mathrm{IL}-1 \beta^{\mathrm{c}}(\mathrm{pg} / \mathrm{ml})$ & $0.11 \pm 0.13$ & $0.45 \pm 1.48$ & 0.76 & 0.26 \\
\hline IL-1ra $(p g / m l)$ & $2,593.2 \pm 3,576.4$ & $2,086.3 \pm 5,507.0$ & 0.03 & 0.10 \\
\hline MMP-1 (ng/ml) & $640.07 \pm 81.58$ & $394.06 \pm 667.06$ & 0.27 & 0.35 \\
\hline MMP-3 (ng/ml) & $4,017.2 \pm 4,576.41$ & $2,532.80 \pm 3,066.43$ & 0.56 & 0.43 \\
\hline$M M P-9(n g / m l)$ & $30.99 \pm 35.96$ & $6.94 \pm 10.30$ & 0.01 & 1.07 \\
\hline NTX-I (nM BCE) & $30.3 \pm 7.9$ & $22.7 \pm 7.1$ & 0.055 & 0.97 \\
\hline TSG-6 (U) & $286.4 \pm 165.7$ & $260.1 \pm 157.3$ & 0.83 & 0.11 \\
\hline
\end{tabular}

${ }^{\text {a }}$ Statistically significant differences denoted with bold and italics font.

${ }^{\mathrm{b}}$ Number of patients in the corticosteroid or placebo group from the original randomized trial.

${ }^{c}$ There was also no difference in the number of samples below LLOD between groups.

$<$ Pass $=3 / 6$ versus $\geq$ PASS $=8 / 16, \mathrm{p}>0.99$.

$\mathrm{d} \mathrm{u}=$ urinary, the remaining biomarkers were measured in synovial fluid. Urinary CTX-II normalized to creatinine level $(\mu \mathrm{g} / \mathrm{mmol})$.

${ }^{\mathrm{e}}$ Cohen's d effect sizes calculations were also performed in order to identify potentially clinically-meaningful findings within these pilot data, with $\mathrm{d}>0.80$ considered a large effect size.

The current pilot results support the previously described cascade of increased IL- $1 \alpha$ stimulating the production of matrix metalloproteinases thereby reducing proteoglycan content and altering cartilage mechanical properties. Previous studies have demonstrated that ACL injury triggers a biochemical cascade that worsens over the first 4-6 weeks after injury [2-4]. Inflammation is initiated early by the injury-related hemarthrosis and subsequent intra-articular pathogenic processes at the time of injury, including the downregulation of proteoglycan synthesis and upregulation of matrix metalloproteinases [29-31]. It is widely accepted that IL-1 and IL-1ra are critical in the regulation of the pathological processes involved in joint tissue breakdown $[32,33]$. Synovial fluid IL-1 levels are elevated in patients with ACL rupture and correlate with severity of chondral damage [34]. Synovial fluid levels of the chondroprotective IL-1ra cytokine are reported to decrease significantly after ACL injury, resulting in relatively unopposed activity of IL1 [1]. Additionally, levels of IL-1ra decrease as the severity of chondral damage is increased [34]. The superficial cartilage layers have been shown to be more susceptible to IL-1 induced damage than deeper layers in vitro [35]. Porcine cartilage explants have been demonstrated to be more sensitive to the chondrodegenerative effects of IL-1 $\alpha$ than IL-1 $\beta$ [36].
The current pilot results suggest that initial biochemical changes after injury may be prognostic of long-term consequences of injury of the knee that are not mitigated by surgical stabilization alone. Inflammatory cytokine and degradative enzyme concentrations on the day of ACL reconstruction have been previously shown to correlate with cartilage changes on MRI during the first three years after surgery [37], and the current results further demonstrate a connection between cytokine and degradative enzyme concentrations and the potential progression of posttraumatic OA. In the current study, both IL- $1 \alpha$ and MMP-9 concentrations on the day of surgery were significantly higher for patients that failed to postoperatively achieve the KOOS QOL-based PASS compared to those with better clinical outcomes. In addition to increased IL-la and MMP-9 concentrations, biomarkers of bone and cartilage turnover (sf NTX-I, $\mathrm{d}=0.97$ and uCTX-II, $d=0.99$ ) were also increased for patients that failed to achieve KOOS QOL-based PASS. This overall state of upregulated catabolism and inflammation may lead to recurrent effusions and/or persistent synovitis which also appears to potentially have long-term implications for knee health.

In addition to playing a contributing role in the progression of structural posttraumatic OA changes [37], increased 
TABLE 4: Comparison of inflammatory cytokines and chondrodegenerative markers (mean \pm standard deviation) evaluated on the day of ACL reconstruction between patients with IKDC scores above and below the PASS threshold of 75.9 points.

\begin{tabular}{|c|c|c|c|c|}
\hline Biomarker & $<$ PASS & $\geq$ PASS & $\mathbf{p}^{\mathrm{a}}$ & $d^{e}$ \\
\hline $\mathrm{N}$ & 9 & 13 & - & - \\
\hline Female/Male (n) & $5 / 4$ & $4 / 9$ & 0.38 & - \\
\hline Steroid/Placebo ${ }^{\mathrm{b}}$ (n) & $6 / 3$ & $10 / 3$ & 0.66 & - \\
\hline Age (years) & $18.9 \pm 3.5$ & $19.9 \pm 4.6$ & 0.82 & - \\
\hline $\operatorname{BMI}\left(\mathrm{kg} / \mathrm{m}^{2}\right)$ & $24.1 \pm 3.9$ & $24.2 \pm 3.5$ & 0.84 & - \\
\hline Graft (BTB/Ham) & $8 / 1$ & $10 / 3$ & 0.62 & - \\
\hline Medial meniscus injury & 7 & 7 & 0.38 & - \\
\hline Lateral meniscus injury & 4 & 7 & $>0.99$ & - \\
\hline Bone bruise volume $\left(\mathrm{mm}^{3}\right)$ & $9.19 \pm 7.94$ & $10.94 \pm 10.18$ & 0.67 & 0.17 \\
\hline COMP $(\mu \mathrm{g} / \mathrm{ml})$ & $30.9 \pm 10.2$ & $41.9 \pm 14.3$ & 0.10 & 0.81 \\
\hline CTX-II (ng/ml) & $1.16 \pm 0.72$ & $1.79 \pm 2.16$ & 0.92 & 0.37 \\
\hline uCTX-II ${ }^{\mathrm{d}}(\mu \mathrm{g} / \mathrm{mmol})$ & $3.92 \pm 4.08$ & $2.91 \pm 2.77$ & 0.62 & 0.30 \\
\hline $\mathrm{sGAG}(\mu \mathrm{g} / \mathrm{ml})$ & $199.5 \pm 70.1$ & $275.8 \pm 183.2$ & 0.82 & 0.51 \\
\hline$I L-1 \alpha(p g / m l)$ & $5.48 \pm 4.56$ & $3.29 \pm 5.80$ & 0.02 & 0.41 \\
\hline $\mathrm{IL}-1 \beta^{\mathrm{c}}(\mathrm{pg} / \mathrm{ml})$ & $0.08 \pm 0.09$ & $0.55 \pm 1.64$ & 0.60 & 0.37 \\
\hline IL-1ra (pg/ml) & $1,899.1 \pm 3,014.8$ & $2,621.5 \pm 6,332.1$ & 0.057 & 0.11 \\
\hline MMP-1 (ng/ml) & $597.22 \pm 689.63$ & $366.96 \pm 715.61$ & 0.10 & 0.33 \\
\hline MMP-3 (ng/ml) & $3,217.23 \pm 4,074.67$ & $2,744.09 \pm 3,180.20$ & 0.87 & 0.14 \\
\hline MMP-9 (ng/ml) & $21.85 \pm 31.59$ & $7.71 \pm 11.31$ & 0.10 & 0.63 \\
\hline NTX-I (nM BCE) & $26.6 \pm 8.6$ & $23.5 \pm 7.5$ & 0.33 & 0.40 \\
\hline TSG-6 (U) & $260.9 \pm 196.8$ & $271.7 \pm 129.3$ & 0.53 & 0.40 \\
\hline
\end{tabular}

${ }^{a}$ Statistically significant differences denoted with bold and italics font.

${ }^{\mathrm{b}}$ Number of patients in the corticosteroid or placebo group from the original randomized trial.

${ }^{c}$ There was also no difference in the number of samples below LLOD between groups.

$<$ Pass $=5 / 9$ ersus $\geq$ PASS $=6 / 13, \mathrm{p}>0.99$.

${ }^{\mathrm{d}} \mathrm{u}=$ urinary, the remaining biomarkers were measured in synovial fluid. Urinary CTX-II normalized to creatinine level $(\mu \mathrm{g} / \mathrm{mmol})$.

${ }^{\mathrm{e}}$ Cohen's d effect sizes calculations were also performed in order to identify potentially clinically-meaningful findings within these pilot data, with $\mathrm{d}>0.80$ considered a large effect size.

inflammatory cytokine and degradative enzyme concentrations on the day of ACL reconstruction also appear to affect postoperative patient-reported outcomes. In OA knees, nerve growth factor expression in the synovium has been shown to modulate pain by increasing local nociceptor sensitization $[38,39]$. Nerve growth factor expression is increased in the synovium of OA knees [40], and by regulating nerve growth factor expression, proinflammatory cytokine IL-1 and other degradative enzymes may play an integral role in modulating knee pain [40]. While attention is given the potential role of the innate immune response on cartilage degradation and the progression of OA [41], it should be noted that, after adjusting for age, peripheral cytokine concentrations are similar between patients with knee $\mathrm{OA}$ and fibromyalgia [42]. Hypersensitivity and nociceptor sensitization secondary to increased proinflammatory cytokine concentrations may then explain the increased IL- $1 \alpha$ and MMP-9 concentrations for those that failed to achieve a Patient Acceptable Symptom State in the current study.

Early anticatabolic and/or anti-inflammatory interventions after ACL injury or reconstruction may need to be further investigated as adjunctive treatment strategies to mitigate both the structural changes and symptoms of posttraumatic OA. CTX-II has been reported to be predictive of OA progression based on both radiographic and arthroscopic evidence [43-45]. Particularly relevant to the current results suggesting a link between CTX-II concentrations and patient-reported outcomes, Ishijima et al. reported that urinary CTX-II was associated with concurrent OA-related pain [46]. The association between CTX-II, pain, and inferior outcomes may be tied to the combination of both changes to the articular cartilage and to bone metabolism. Garnero et al. first reported that urinary CTX-II concentrations in early OA patients were predictive of bone marrow lesion progression [47]. More recently, in a cluster analysis of a wide spectrum of OA-related biomarkers, van Spil reported that CTX-II tended to cluster with other biomarkers associated with bone metabolism [48]. Bone marrow lesions are common following ACL injury [26, 49], and many resolve over time [50]. However, subjective symptoms of early OA are more common six years after ACL reconstruction for those noted to have local articular cartilage damage combined with bone marrow lesions at the time of surgery [26]. The size and progression of bone marrow lesions have been previously linked to OA-related pain as the subchondral bone is rich with nociceptors whereas the articular cartilage is not [5153]. Taken together with our data, links between CTX-II, NTX-I, IL- $1 \alpha$, pain, and both cartilage and bone metabolism 
may all contribute to KOOS QOL and IKDC outcomes at 2 years.

This study had several limitations. First and foremost, the results of this preliminary work are based on a small sample size and additional studies are necessary to confirm these results. Second, some patients had received corticosteroid injections prior to surgery which could have influenced biomarker concentrations on the day of surgery. As mentioned previously, KOOS QOL and IKDC scores did not differ between patients treated with preoperative intra-articular corticosteroid and the placebo group, and the surgical intervention and postoperative rehabilitation and medications did not differ between groups and no between-group differences were noted in either postoperative KOOS QOL or IKDC scores. Third, the prevalence of patients with postoperative KOOS QOL and IKDC scores below the PASS threshold was higher in the current study than those originally reported by Muller et al. [18]. This may be due, in part, to the differences in patient populations between the two studies. In the current study, all patients were injured during athletic participation with an average age at the time of surgery of 19.5 years compared to 26.1 in the study by Muller et al. [18]. The lingering effects of athletic injuries have been reported to negatively impact quality of life years after competition has ended, and both physical and mental aspects of function are significantly lower in former collegiate athletes compared to college attendees who did not participate in sport [54]. It remains unclear if the increased prevalence of patients with inferior patient-reported outcomes in the current study was perhaps due to the strict inclusion criteria of athletic ACL injuries. Fourth, while inferior patient-reported outcome scores have been associated with early OA changes, postoperative imaging or biomarker evidence of $\mathrm{OA}$ will be necessary to establish the potential connection between biomarkers on the day of surgery, KOOS QOL, IKDC scores, and evidence of structural features of knee OA. Finally, future work may require more sensitive methods to quantify synovial fluid IL-1 $\beta$ concentrations as $50 \%$ of the samples in the current study had values below the lower limits of detection.

In conclusion, the results of this preliminary investigation demonstrated that higher inflammatory cytokine activity (IL$1 \alpha$ ) and a trend towards greater bone and cartilage collagen degradation (NTX-I and uCTX-II) at the time of surgery were associated with failure to achieve an acceptable symptom state two years after ACL reconstruction. These data suggest that initial biochemical changes after injury may be prognostic of long-term consequences of ACL injury that are not mitigated by surgical stabilization alone; however, due to the small sample size these data should be used for hypothesis generation and more definitive randomized clinical trials are necessary.

\section{Data Availability}

The datasets used to support the findings of this study are restricted by the University of Kentucky Institutional Review Board in order to protect patient privacy. Data are available from the corresponding author for researchers who meet the criteria for access to confidential data.

\section{Disclosure}

This study was conducted at the University of Kentucky and all analyses of biological specimens were performed at Duke University. The content is solely the responsibility of the authors and does not necessarily represent the official views of the National Institutes of Health or the Arthroscopy Association of North America. This study was presented in part at the 2018 International Cartilage Regeneration \& Joint Preservation Society World Congress (ID 9981).

\section{Conflicts of Interest}

The authors have no conflicts of interest related to this work.

\section{Acknowledgments}

This study received funding from The Arthritis Foundation of America and the Arthroscopy Association of North America. Research reported in this publication was supported by the National Institute of Arthritis and Musculoskeletal and Skin Diseases of the National Institutes of Health under Award no. 5K23AR060275. Data collection and study administration was supported by the University of Kentucky CTSA Award (UL1TR000117).

\section{References}

[1] M. Cameron, A. Buchgraber, H. Passler et al., "The natural history of the anterior cruciate ligament-deficient knee. Changes in synovial fluid cytokine and keratan sulfate concentrations," The American Journal of Sports Medicine, vol. 25, no. 6, pp. 751-754, 1997.

[2] J. B. Catterall, T. V. Stabler, C. R. Flannery, and V. B. Kraus, "Changes in serum and synovial fluid biomarkers after acute injury (NCT00332254)," Arthritis Research \& Therapy, vol. 12, no. 6, article R229, 2010.

[3] C. Lattermann, C. A. Jacobs, M. Proffitt Bunnell et al., "A Multicenter Study of Early Anti-inflammatory Treatment in Patients With Acute Anterior Cruciate Ligament Tear," The American Journal of Sports Medicine, vol. 45, no. 2, pp. 325-333, 2016.

[4] C. Lattermann, C. A. Jacobs, M. P. Bunnell et al., "Logistical challenges and design considerations for studies using acute anterior cruciate ligament injury as a potential model for early posttraumatic osteoarthritis," Journal of Orthopaedic Research, vol. 35, no. 3, pp. 641-650, 2017.

[5] L. S. Lohmander, P. M. Englund, L. L. Dahl, and E. M. Roos, "The long-term consequence of anterior cruciate ligament and meniscus injuries: osteoarthritis," The American Journal of Sports Medicine, vol. 35, no. 10, pp. 1756-1769, 2007.

[6] L. S. Lohmander and H. Roos, "Knee ligament injury, surgery and osteoarthrosis: Truth or consequences?" Acta Orthopaedica, vol. 65, no. 6, pp. 605-609, 1994.

[7] B. E. Øiestad, L. Engebretsen, K. Storheim, and M. A. Risberg, "Knee osteoarthritis after anterior cruciate ligament injury: a systematic review," The American Journal of Sports Medicine, vol. 37, no. 7, pp. 1434-1443, 2009. 
[8] D. Hunter, A. Guermazi, G. Lo et al., "Evoluation of semiquantitative whole joint assement of knee OA: MOAKS (MRI Osteoarthritis Knee Score)," Osteoarthritis and Cartilage, vol. 19, no. 8, pp. 990-1002, 2011.

[9] F. P. Luyten, M. Denti, G. Filardo, E. Kon, and L. Engebretsen, "Definition and classification of early osteoarthritis of the knee," Knee Surgery, Sports Traumatology, Arthroscopy, vol. 20, no. 3, pp. 401-406, 2012.

[10] H. Madry, E. Kon, V. Condello et al., "Early osteoarthritis of the knee," Knee Surgery, Sports Traumatology, Arthroscopy, vol. 24, no. 6, pp. 1753-1762, 2016.

[11] R. W. Wright and T. M. Group, "Osteoarthritis classification scales: interobserver reliability and arthroscopic correlation," Journal of Bone and Joint Surgery, vol. 96, no. 14, pp. 1145-1151, 2014.

[12] V. B. Kraus, F. J. Blanco, M. Englund et al., "OARSI Clinical Trials Recommendations: Soluble biomarker assessments in clinical trials in osteoarthritis," Osteoarthritis and Cartilage, vol. 23, no. 5, pp. 686-697, 2015.

[13] V. B. Kraus, J. E. Collins, D. Hargrove et al., "Predictive validity of biochemical biomarkers in knee osteoarthritis: data from the FNIH OA Biomarkers Consortium," Annals of the Rheumatic Diseases, vol. 76, no. 1, pp. 186-195, 2016.

[14] S. J. Svoboda, T. M. Harvey, B. D. Owens, W. F. Brechue, P. M. Tarwater, and K. L. Cameron, "Changes in serum biomarkers of cartilage turnover after anterior cruciate ligament injury," The American Journal of Sports Medicine, vol. 41, no. 9, pp. 21082116, 2013.

[15] M. Dougados, "It's good to feel better but it's better to feel good," The Journal of Rheumatology, vol. 32, no. 1, pp. 1-2, 2005.

[16] J. C. Keurentjes, F. R. Van Tol, M. Fiocco et al., "Patient acceptable symptom states after total hip or knee replacement at midterm follow-up," Bone \& Joint Research, vol. 3, no. 1, pp. 7-13, 2014.

[17] T. K. Kvien, T. Heiberg, and K. B. Hagen, "Minimal clincally important improvement/difference (MCII/MCID) and the patient acceptable symptom state (PASS): what do these concepts mean?" Annals of the Rheumatic Diseases, vol. 66, no. 3, pp. 4041, 2007.

[18] B. Muller, M. A. Yabroudi, A. Lynch et al., "Defining Thresholds for the Patient Acceptable Symptom State for the IKDC Subjective Knee Form and KOOS for Patients Who Underwent ACL Reconstruction," The American Journal of Sports Medicine, vol. 44, no. 11, pp. 2820-2826, 2016.

[19] R. C. Glattes, K. P. Spindler, G. M. Blanchard, M. T. Rohmiller, E. C. McCarty, and J. Block, "A simple, accurate method to confirm placement of intra-articular knee injection," The American Journal of Sports Medicine, vol. 32, no. 4, pp. 1029-1031, 2004.

[20] C. Chou, D. Attarian, H. Wisniewski, P. Band, and V. Kraus, "TSG-6 - a double-edged sword for osteoarthritis (OA)," Osteoarthritis and Cartilage, vol. 26, no. 2, pp. 245-254, 2018.

[21] M. Englund, E. M. Roos, and L. S. Lohmander, "Impact of type of meniscal tear on radiographic and symptomatic knee osteoarthritis: A sixteen-year followup of meniscectomy with matched controls," Arthritis \& Rheumatology, vol. 48, no. 8, pp. 2178-2187, 2003.

[22] S. R. Filbay, I. N. Ackerman, T. G. Russell, E. M. Macri, and K. M. Crossley, "Health-related quality of life after anterior cruciate ligament reconstruction: a systematic review," The American Journal of Sports Medicine, vol. 42, no. 5, pp. 1247-1255, 2014.
[23] J. J. Irrgang, A. F. Anderson, A. L. Boland et al., "Development and validation of the international knee documentation committee subjective knee form," The American Journal of Sports Medicine, vol. 29, no. 5, pp. 600-613, 2001.

[24] E. M. Roos, H. P. Roos, L. S. Lohmander, C. Ekdahl, and B. D. Beynnon, "Knee Injury and Osteoarthritis Outcome Score (KOOS)-development of a self-administered outcome measure," Journal of Orthopaedic \& Sports Physical Therapy, vol. 28, no. 2, pp. 88-96, 1998.

[25] D. Schiphof, J. Waarsing, E. Oei, and S. Bierma-Zeinstra, "KOOS subscale and individual items associate with onset of structural knee osteoarthritis within five years," Osteoarthritis and Cartilage, vol. 24, p. S438, 2016.

[26] C. Lattermann, C. A. Jacobs, E. K. Reinke et al., "Are Bone Bruise Characteristics and Articular Cartilage Pathology Associated with Inferior Outcomes 2 and 6 Years After Anterior Cruciate Ligament Reconstruction?" Cartilage, vol. 8, no. 2, pp. 139-145, 2017.

[27] F. W. Roemer and K. Bohndorf, "Long-term osseous sequelae after acute trauma of the knee joint evaluated by MRI," Skeletal Radiology, vol. 31, no. 11, pp. 615-623, 2002.

[28] J. Cohen, Statistical power analysis for the behavioral sciences, vol. 2nd, Lawrence Earlbaum Associates, Hillsdale, NJ, 1988.

[29] S. Chandrasekhar, A. K. Harvey, and P. S. Hrubey, "IntraArticular Administration of Interleukin-1 Causes Prolonged Suppression of Cartilage Proteoglycan Synthesis in Rats," Matrix Biology, vol. 12, no. 1, pp. 1-10, 1992.

[30] M. B. Goldring, J. Birkhead, L. J. Sandell, T. Kimura, and S. M. Krane, "Interleukin 1 suppresses expression of cartilage-specific types II and IX collagens and increases types I and III collagens in human chondrocytes," The Journal of Clinical Investigation, vol. 82, no. 6, pp. 2026-2037, 1988.

[31] C. Lundberg, I. Åsberg, M. Lonescu, A. Reiner, G. Smedegård, and A. Robin Poole, "Changes in cartilage proteoglycan aggrecan after intra-articular injection of interleukin-1 in rabbits: Studies of synovial fluid and articular cartilage," Annals of the Rheumatic Diseases, vol. 55, no. 8, pp. 525-534, 1996.

[32] C. Jacques, M. Gosset, F. Berenbaum, and C. Gabay, “The Role of IL-1 and IL-1Ra in Joint Inflammation and Cartilage Degradation," Vitamins \& Hormones, vol. 74, pp. 371-403, 2006.

[33] M. Lotz, "Cytokines in cartilage injury and repair," Clinical Orthopaedics and Related Research, no. 391, pp. S108-S115, 2001.

[34] P. H. Marks and M. L. C. Donaldson, "Inflammatory cytokine profiles associated with chondral damage in the anterior cruciate ligament-deficient knee," Arthroscopy: The Journal of Arthroscopic and Related Surgery, vol. 21, no. 11, pp. 1342-1347, 2005.

[35] H. J. Hauselmann, J. Flechtenmacher, and L. Michal, “The superficial layer of human articular cartilage is more susceptible to interleukin-1-induced damage than the deeper layers," Arthritis \& Rheumatism, vol. 39, no. 3, pp. 478-488, 1996.

[36] A. L. McNulty, N. E. Rothfusz, H. A. Leddy, and F. Guilak, "Synovial fluid concentrations and relative potency of interleukin-1 alpha and beta in cartilage and meniscus degradation," Journal of Orthopaedic Research, vol. 31, no. 7, pp. 1039-1045, 2013.

[37] K. Amano, J. L. Huebner, T. V. Stabler et al., "Synovial Fluid Profile at the Time of Anterior Cruciate Ligament Reconstruction and Its Association With Cartilage Matrix Composition 3 Years After Surgery," The American Journal of Sports Medicine, vol. 46, no. 4, pp. 890-899, 2018. 
[38] S. Pezet and S. B. McMahon, "Neurotrophins: mediators and modulators of pain," Annual Review of Neuroscience, vol. 29, pp. 507-538, 2006.

[39] C. J. Woolf, B. Safieh-Garabedian, Q.-P. Ma, P. Crilly, and J. Winter, "Nerve growth factor contributes to the generation of inflammatory sensory hypersensitivity," Neuroscience, vol. 62, no. 2, pp. 327-331, 1994.

[40] S. Takano, K. Uchida, G. Inoue et al., "Nerve growth factor regulation and production by macrophages in osteoarthritic synovium," Clinical \& Experimental Immunology, vol. 190, no. 2, pp. 235-243, 2017.

[41] F. Berenbaum, "Osteoarthritis as an inflammatory disease (osteoarthritis is not osteoarthrosis!)," Osteoarthritis and Cartilage, vol. 21, no. 1, pp. 16-21, 2013.

[42] M. Imamura, R. A. Targino, and W. T. Hsing, "Concentrations of cytokines in pateints with osteoarthritis of the knee and fibromyalgia," Clinical Interventions in Aging, vol. 9, pp. 939944, 2014.

[43] P. Garnero, X. Ayral, J.-C. Rousseau et al., "Uncoupling of type II collagen synthesis and degradation predicts progression of joint damage in patients with knee osteoarthritis," Arthritis \& Rheumatology, vol. 46, no. 10, pp. 2613-2624, 2002.

[44] J. Kumm, A. Tamm, M. Lintrop, and A. Tamm, "The value of cartilage biomarkers in progressive knee osteoarthritis: crosssectional and 6-year follow-up study in middle-aged subjects," Rheumatology International, vol. 33, no. 4, pp. 903-911, 2013.

[45] M. Reijman, J. M. Hazes, S. M. Bierma-Zeinstra et al., "A new marker for osteoarthritis: Cross-sectional and longitudinal approach," Arthritis \& Rheumatism, vol. 50, no. 8, pp. 24712478, 2004.

[46] M. Ishijima, T. Watari, K. Naito et al., "Relationships between biomarkers of cartilage, bone, synovial metabolism and knee pain provide insights into the origins of pain in early knee osteoarthritis," Arthritis Research \& Therapy, vol. 13, article R22, 2011.

[47] P. Garnero, C. Peterfy, S. Zaim, and M. Schoenharting, "Bone marrow abnormalities on magnetic resonance imaging are associated with type II collagen degradation in knee osteoarthritis: A three-month longitudinal study," Arthritis \& Rheumatology, vol. 52, no. 9, pp. 2822-2829, 2005.

[48] W. E. Van Spil, N. W. D. Jansen, J. W. J. Bijlsma et al., "Clusters within a wide spectrum of biochemical markers for osteoarthritis: Data from CHECK, a large cohort of individuals with very early symptomatic osteoarthritis," Osteoarthritis and Cartilage, vol. 20, no. 7, pp. 745-754, 2012.

[49] H. G. Potter, S. K. Jain, Y. Ma, B. R. Black, S. Fung, and S. Lyman, "Cartilage injury after acute, isolated anterior cruciate ligament tear: immediate and longitudinal effect with clinical/MRI follow-up," The American Journal of Sports Medicine, vol. 40, no. 2, pp. 276-285, 2012.

[50] M. Costa-Paz, D. L. Muscolo, M. Ayerza, A. Makino, and L. Aponte-Tinao, "Magnetic resonance imaging follow-up study of bone bruises associated with anterior cruciate ligament ruptures," Arthroscopy: The Journal of Arthroscopic and Related Surgery, vol. 17, no. 5, pp. 445-449, 2001.

[51] D. T. Felson, C. E. Chaisson, C. L. Hill, and etal., "The association of bone marrow lesions with pain in knee osteoarthritis," Annals of Internal Medicine, vol. 134, pp. 591-593, 2001.

[52] D. T. Felson, S. McLaughlin, J. Goggins et al., "Bone Marrow Edema and Its Relation to Progression of Knee Osteoarthritis," Annals of Internal Medicine, vol. 139, no. 5 I, pp. 330-I33, 2003.
[53] D. T. Felson, J. Niu, A. Guermazi et al., "Correlation of the development of knee pain with enlarging bone marrow lesions on magnetic resonance imaging," Arthritis \& Rheumatology, vol. 56, no. 9, pp. 2986-2992, 2007.

[54] J. E. Simon and C. L. Docherty, "Current health-related quality of life is lower in former division i collegiate athletes than in non-collegiate athletes," The American Journal of Sports Medicine, vol. 42, no. 2, pp. 423-429, 2014. 


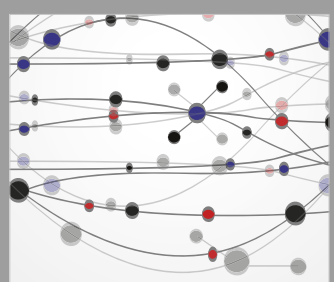

The Scientific World Journal
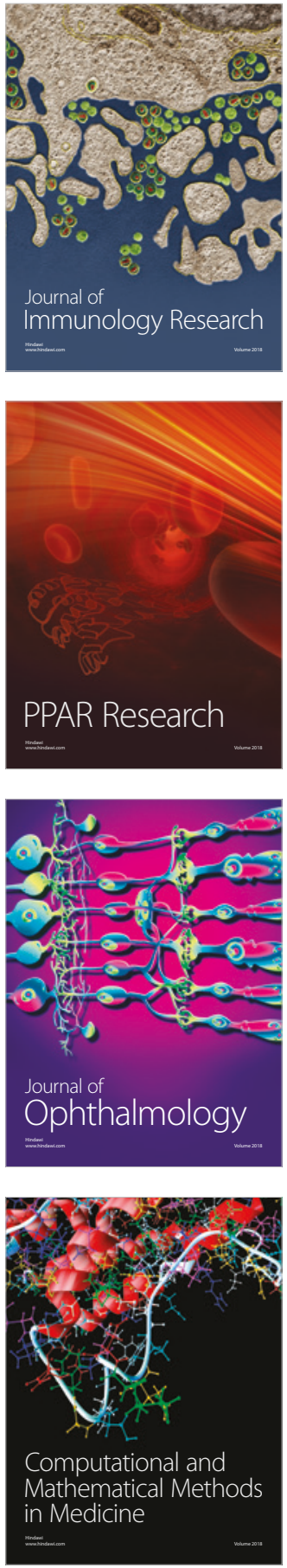

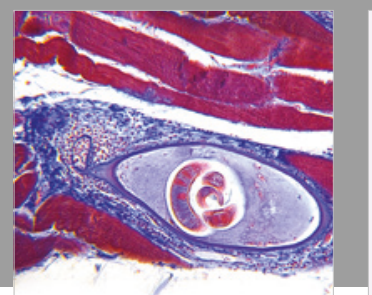

Gastroenterology Research and Practice

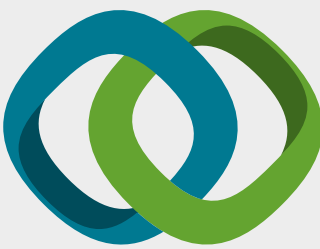

\section{Hindawi}

Submit your manuscripts at

www.hindawi.com
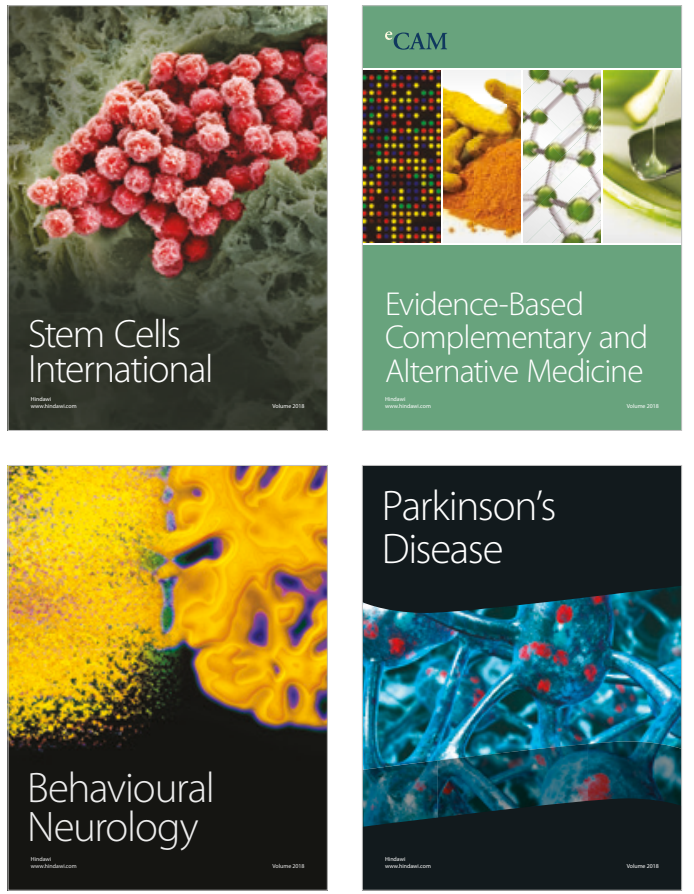

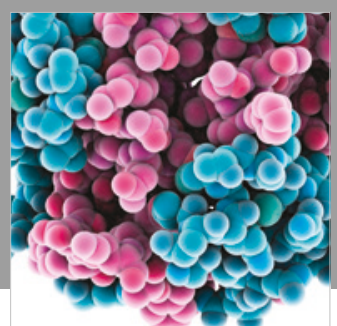

ournal of

Diabetes Research

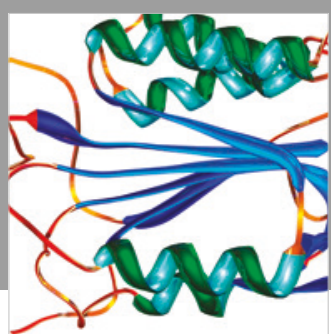

Disease Markers
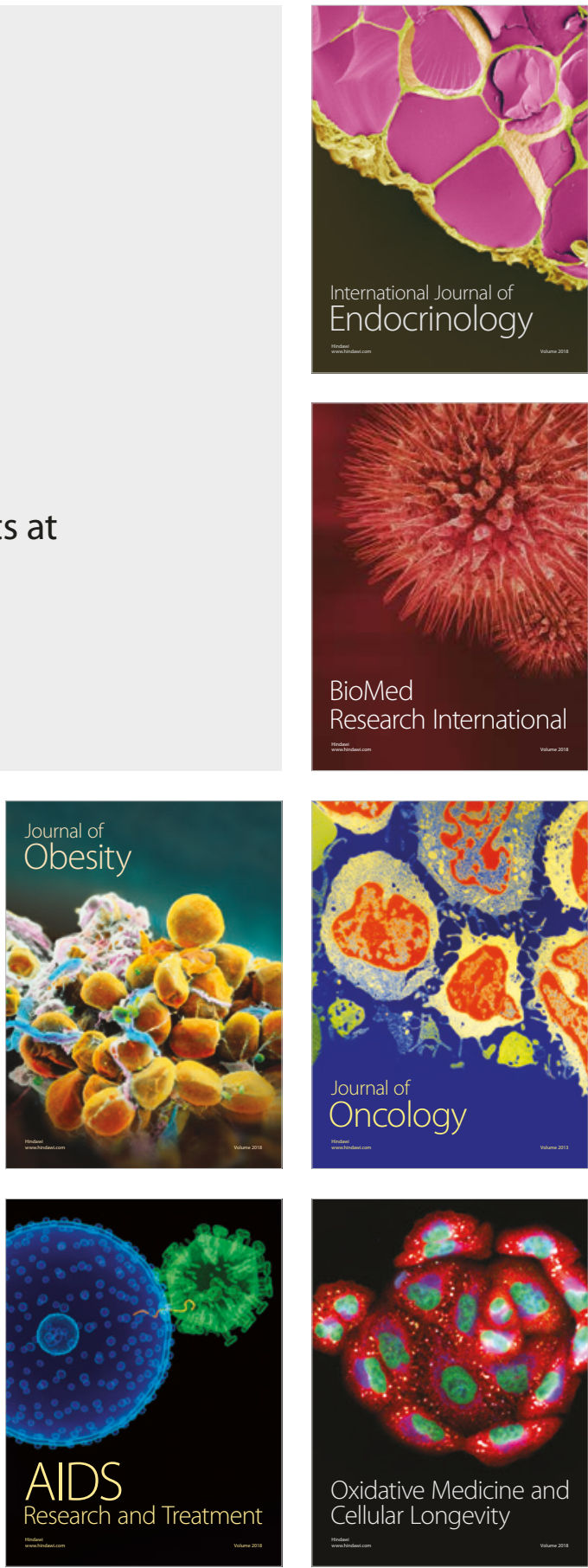